\title{
FutureJournal
}

\section{Gestão Empresarial e Inovação: uma Análise sobre os Determinantes da Inovação em Micro e Pequenas Empresas do Setor de Alimentos e Bebidas}

\author{
Renata Braga Berenguer de Vasconcelos \\ Graduação em Administração pela Universidade Federal de Pernambuco, UFPE, Brasil. \\ renata_berenguer@hotmail.com
}

Paula Roberta Callado Bezerra de Mello

Doutoranda em Administração pela Universidade Federal de Pernambuco, UFPE, Brasil. www.callado@gmail.com

\section{Francisco Vicente Sales Melo}

Professor Adjunto do Departamento de Ciências Administrativas da Universidade Federal de Pernambuco, UFPE, Brasil.

vicsmelo@gmail.com

\section{RESUMO}

A capacidade inovadora desenvolvida por micro e pequenas empresas (MPEs) pode ser determinante para o sucesso delas no mercado. Neste artigo, o objetivo é analisar a relação entre o grau de inovação e o nível de gestão de MPES do setor de alimentos e bebidas. Para tanto, a perspectiva holística de inovação proposta pelo radar da inovação desenvolvido por Sawhney, Wolcott e Arroniz (2006) foi adotada tendo-se como base as oito dimensões do modelo de excelência em gestão. Por meio de regressão linear múltipla, foi possível verificar quais dimensões de gestão impactam o grau de inovação dessas empresas. Os resultados indicam que as dimensões clientes, sociedade, informação e conhecimento e processos são as principais determinantes da inovação nas micro e pequenas empresas do setor de alimentos e bebidas estudadas. $O$ estudo contribui para literatura ao identificar que, apesar de todas as dimensões do modelo de excelência em gestão serem indicadas como relevantes no processo de inovação das MPES, para o mercado analisado, elas tendem a sofrer variações, sendo mais relevante a dimensão informação e conhecimento.

PALAVRAS-CHAVE: Grau de inovação. Grau de gestão. Radar de inovação. Micro e pequenas empresas. 


\section{Business Management and Innovation: an Analysis of the} Determinants of Innovation in Micro and Small Enterprises in the Food and Beverage Sector

\section{ABSTRACT}

The innovative capacity developed by Micro and Small Enterprises (MSEs) may be crucial to their success the market. This article aims to analyze the relationship between degree of innovation and management level in of MSEs the food and beverage industry. To this end, the holistic perspective of innovation proposed by the innovation radar developed by Sawhney, Wolcott and Arroniz (2006) was adopted taking as basis the eight dimensions of the excellence in management model. Through multiple linear regression, it was possible to verify which management dimensions impact the degree of innovation of these firms. The results indicate that the dimensions customers, society, information and knowledge and processes are the main determinants of innovation in micro and small enterprises analyzed in the food and beverage sector. The study brings a contribution to the literature in identifying that despite all the dimensions of the excellence model in management being indicated as relevant in the MSEs innovation process, for the analyzed market, these tend to be varied, and the most relevant dimension is information and knowledge.

KEY-WORDS: Popular participation. Civil society. Public management. Local power. Accountability. 


\section{INTRODUÇÃO}

A pesquisa sobre inovação em serviços tem se ampliando como campo de pesquisa, afinal o setor de serviços ganhou nas ultimas décadas um papel central nas economias dos países, chegando a responder por parcela importante do Produto Interno Bruto (PIB). Percebeu-se, entretanto, que poucos estudos abordam as determinantes de gestão para que uma empresa prestadora de serviços invista em inovação. Nesse sentido, conforme destacado por Bignetti (2006), os trabalhos sobre gestão da Tecnologia e Inovação, dividem-se em quatro abordagens principais: organização e inovação, estudos sobre $P \& D$, relações interorganizacionais e desenvolvimento de serviços. Em estudo, Chen, Tsou e Huang (2009) elencam três fatores determinantes para a inovação no setor: colaboração com parceiros externos, orientação para inovação e capacidade de tecnologia (apoiado na infraestrutura, recursos humanos e ativos intangíveis de TI), considerando-se apenas algumas das dimensões de gestão empresarial.

Chen, Podolski e Veeraraghavan (2015) analisaram se a capacidade gerencial afeta positivamente os resultados da inovação em grandes corporações e concluíram que a capacidade pessoal do administrador é um componente essencial para o sucesso inovador de grandes empresas. Embora a gestão de MPEs seja fortemente centrada na figura do gestor, Chen et al. (2015) limitaram-se a analisar a influência de aspectos pessoais do executivo sobre a inovação de grandes empresas, não abordando os processos gerenciais em si.

Com isso, este artigo contribui para a literatura ao ampliar os aspectos de gestão propostos por Chen et al. (2009) e ao analisar diversas dimensões de gestão nas MPEs. Neste objetivo, no estudo examina-se a relação de diversas áreas da administração da empresa sobre os investimentos em inovação em micro e pequenas empresas (MPEs) na região metropolitana do Recife, mais especificamente, sobre o segmento de restaurantes e outros estabelecimentos de serviço de alimentação e bebidas. Estabeleceu-se, então, como objetivo geral: verificar o impacto das 
dimensões de gestão no grau de inovação em MPEs do segmento de alimentos e bebidas da Região Metropolitana do Recife, tendo-se como objetivos específicos: mensurar o grau de inovação (GI), mensurar o nível de gestão segundo as dimensões estudadas e verificar quais variáveis de gestão influenciam o grau de inovação dessas empresas. Parte-se do pressuposto de que há relação positiva entre o grau de inovação da organização e o nível de gestão nas empresas de pequeno porte.

\section{REFERENCIAL TEÓRICO}

\subsection{CONCEITO E TIPOS DE INOVAÇÃO}

Os estudos em administração propõem diversas acepções para o termo inovação, tornando-o um conceito plural e multifacetado. Dada a complexidade do processo de inovação e as diversas formas como ela ocorre na economia, percebeu-se a necessidade de adotar convenções, cujo objetivo foi estabelecer definições operacionais passíveis de serem usadas de forma padronizada em estudos sobre inovação em empresas. Destarte, já em 1990, foi editado pela primeira vez pela Organização para Cooperação e Desenvolvimento Econômico (OCDE) o Manual de Oslo, cujo conteúdo reúne orientações e padronizações de conceitos, metodologias, indicadores e métodos estatísticos sobre inovação. Mais recentemente, em 2005, o documento foi atualizado trazendo como conceito de inovação a "implementação de um produto, seja ele um bem ou serviço, ou um processo, método nas práticas de negócios novos ou significativamente melhorados" (OECD, 2005, p. 55). No que concerne à inovação em serviços, esta foi originalmente compreendida pela literatura como mero resultado de inovação tecnológica ou da produção (Barras, 1986).

A relação entre inovação e gestão tem surgido como importante campo de análise em consequência do papel central da inovação para estratégias competitivas eficazes nas empresas. Observa-se figurar, como abordagem mais comum sobre o tema, a influência que o processo de inovação nas empresas tem sobre os resultados gerenciais. A contribuição aqui proposta, todavia, visa suprir uma lacuna nas pesquisas realizadas 
sobre inovação ao realizar um estudo da ingerência de aspectos da gestão de empresas sobre sua propensão a inovar. Prajogo (2006), por exemplo, investigou a relação entre inovação em produto e processos e desempenho em empresas dos setores industrial e de serviços.

Perin, Sampaio e Hooley (2007), por sua vez, partiram da abordagem Visão Baseada em Recursos (VBR) da empresa, para avaliar o papel que os recursos internos da empresa podem assumir no desempenho de inovação, com ênfase em recursos como a orientação para o mercado, as capacidades gerenciais, a capacidade de conexão com os clientes, os ativos de recursos humanos e a capacidade de inovação. O estudo que inspirou parte da análise aqui proposta envolveu empresas de diversos setores econômicos do mercado nacional, indicou, que, além de tais recursos internos, ativos e habilidades empresariais interferem no desempenho da inovação nas empresas. Importante se faz, portanto, conhecer qual a relação existente entre as diversas dimensões de gestão empresarial que explicam o grau de inovação das empresas no mercado de alimentos.

\subsection{RELAÇÃO ENTRE DIMENSÕES DE GESTÃO E INOVAÇÃO NAS EMPRESAS}

Com foco no desenvolvimento de uma tipologia de inovação, Paswan, D’Souza e Zolfagharian (2009) propõem a divisão de empresas do setor de serviços em sete células: dimensão externa; orientação para o cliente; incertezas no ambiente; incerteza e inovação; orientação de mercado; orientação estratégica; orientação estratégica e inovação, as quais os autores terminam resumindo em incerteza do ambiente, orientação estratégica e orientação para o mercado. A metodologia de análise aqui adotada quanto à gestão empresarial considera essas dimensões e propõe outras novas. No tocante à percepção de incertezas no ambiente, os autores defendem que, quanto maior for a percepção de incertezas ambientais, maior será a percepção de risco e de oportunidades, gerando uma maior propensão a inovações mais profundas e não apenas incrementais. 
A inovação empresarial representa um dos mais importantes motores para o sucesso organizacional de longo prazo (Holmstrom, 1989). Para Bignetti (2006), os estudos acadêmicos sobre inovação frequentemente salientam a importância da inovação para a competitividade. Porter $(1989,1993)$ figura como um dos autores com significativas contribuições sobre vantagem competitiva. destacando as estratégias competitivas de custo e diferenciação e um conjunto de forças competitivas das empresas. Assim como a inovação se apresenta em diversos aspectos, a gestão de uma empresa é realizada em múltiplas dimensões e é a relação entre esses dois processos que se visa analisar a partir da hipótese proposta:

H0: Há relação positiva entre o grau de inovação e o nível de gestão nas empresas de pequeno porte.

Esta hipótese origina-se da constatação de estudos sobre a influência de variáveis internas às empresas e seu desempenho de inovação. Mesmo que não denominadas dimensões de gestão, as relações estabelecidas pelos autores demonstram que a estrutura de gestão exerce um papel importante nas ações de inovação desenvolvidas pela organização. Destaca-se, neste domínio, Quadros (2005), para quem a gestão da inovação exige coordenação e mobilização de recursos, juntamente com os parceiros internos e com parceiros externos (abrangendo aqui desde clientes, a fornecedores, concorrentes, instituições de pesquisa e fomento). Em sua análise, Quadros elaborou o Modelo de Gestão Estratégica da Inovação Tecnológica focando os processos e as rotinas para estruturar a gestão da inovação sob uma perspectiva estratégica.

Apesar das restrições típicas de uma MPE relacionadas a escala, preço e qualidade se comparadas às grandes empresas multinacionais, em seu estudo de caso, Meza (2009) constatou que uma microempresa é capaz de promover inovações em produtos, processos e gestão de pessoas, alinhando os objetivos das estratégias de inovação aos objetivos das estratégias de competição e de mercado. 
A gestão da informação é uma das dimensões concebidas para análise gerencial. Santa, Scavarda, Zaho e Skoko (2011) analisaram como a implementação de inovações em tecnologias de informação (a exemplo de Enterprise Resource Planning - ERP) impacta os resultados operacionais de longo prazo. A literatura também indica que um relevante direcionador do processo de inovação são as necessidades dos consumidores. Portanto, conforme destacado por Panesar e Markeset (2008), mecanismos de feedback dos clientes figuram como atividade vital para o incentivo à inovação no setor de serviços.

Outra dimensão relevante sobre as ações gerenciais das empresas é a política de gestão dos colaboradores, cuja relação com padrões de inovação foi abordada por Souza e Bastos (2009). Ainda, tangenciando a dimensão pessoas e estratégia da taxonomia dos aspectos de gestão adotados no presente trabalho, Gebauer, Krempi, Fleisch e Frideli (2008) observaram haver 15 variáveis que impactam na inovação, dentre as quais o envolvimento dos trabalhadores da linha de frente, a distribuição da informação, a constituição e a educação das equipes, a tecnologia de informação, a organização interna.

Sutthijakra (2011) apresentou uma contribuição para a análise da relação de outra dimensão de gestão com inovação: sociedade. Afinal, observou-se em seu estudo que a adoção de inovações pela implantação de melhores práticas, considerando-se os aspectos contextuais, reduz as tensões entre a delimitação de padrões gerais e a adaptação local.

Pesquisas sobre inovação indicam o importante papel dos processos de gestão das empresas sobre sua atividade inovadora. Neely e Hii (1998, p. 23) afirmam que a capacidade de inovação de uma companhia pode ser entendida como seu potencial para gerar resultados inovadores. Potencial que depende, segundo os autores, da inter-relação sinérgica da cultura da empresa, dos processos internos e do ambiente externo.

Quanto ao aspecto de resultados econômicos da empresa, outro nível de gestão aqui analisado, é possível citar o trabalho de Cainelli, Evangelista e Savona (2006). Os autores analisaram a influência de resultados econômicos em empresas do setor de serviços sobre ações de inovação, concluindo por uma relação positiva entre as variáveis, de modo a 
observar-se uma relação de autorreforço da dimensão econômica da empresa através da inovação. Na mesma linha de análise, Canepa e Stonemany (2007) investigaram que aspectos financeiros impactam significativamente a inovação promovida em empresas. Os autores também se basearam em dados de 1998-2000 da Community Innovation Survey e perceberam uma maior sensibilidade nessa relação para pequenas empresas e setores de tecnologia. Concluíram ainda que os resultados econômicos podem ser afetados por limites financeiros ou de liquidez cujo impacto alcança as atividades de inovação das companhias.

Como se pode ver, os autores mencionados demonstram que a inovação é afetada por diferentes variáveis internas à empresa, aqui denominadas variáveis de gestão. Todavia, grande parte dos trabalhos realizados enfocam grandes organizações, cuja estrutura de gestão se encontra definida e consolidada. É necessário, portanto, lançar o olhar às micro e pequenas empresas, entendendo suas diferenças e limitações a fim de verificar as relações aí estabelecidas.

\subsection{INOVAÇÃO NAS MICRO E PEQUENAS EMPRESAS}

$\mathrm{Na}$ literatura sobre inovação, desenvolve-se um debate teórico sobre a relação entre a geração de inovação e o tamanho da empresa. A expressividade das grandes empresas nos investimentos em inovação é bastante importante, principalmente, quando vistos em termos de Pesquisa e Desenvolvimento $(P \& D)$, número de patentes e grau de intangibilidade. Segundo destacado por Grynzpan (2007), dados da Associação Nacional de Pesquisa e Desenvolvimento das Empresas Inovadoras (Anpei) indicam que quase $91,8 \%$ dos investimentos privados em P\&D foram realizados por grandes e médias empresas.

De acordo com pesquisa efetuada pelo Serviço Brasileiro de Apoio às Micro e Pequenas Empresas (Sebrae) com mais de 34 mil empreendimentos, as MPEs realizaram mais de 85 mil ações de inovação no ano de 2013, correspondendo em sua maioria a criação de produtos, novos meios de fabricação ou distribuição de bens e novas formas de prestação de serviços (Exame, 2013). 
Partindo da hipótese schumpeteriana, existem grandes diferenças entre as inovações praticadas pelas empresas grandes (mais estruturadas) e pelas pequenas (fortemente baseadas no empreendedorismo), de modo que a inovação tende a crescer com o tamanho da empresa. Póvoa e Monsueto (2012), por exemplo, correlacionaram o tamanho da empresa com sua propensão a inovar, em termos de acesso a fontes externas de inovação (tais como universidades e centro de pesquisas) e verificaram que há controvérsias na hipótese schumpeteriana. Apesar do imenso debate teórico corroborado por tantos outros autores, a relação entre inovação e tamanho da empresa não é tão clara. Não há evidências de uma associação positiva geral entre a inovação e a estrutura do mercado ou o tamanho da empresa, apesar de existirem circunstâncias em que há uma associação positiva (Symeonidis, 1996).

Há de se considerar também que os resultados encontrados podem ser afetados pela variável utilizada para mensurar inovação. Indicadores tradicionais, como investimentos em $P \& D$ e número de patentes, não são adequados para as MPEs (Bachmann \& Destefani, 2008). Ademais, como ponderam Sawhney, Wolcott e Arroniz (2006), as inovações ocorridas nas MPEs, na maioria das vezes, não são fruto dos investimentos em $P \& D$, mas são trazidas por fornecedores, concorrentes ou colaboradores e raramente são objeto de patenteamento. Dessa forma, para avaliar o grau de inovação nas micro e pequenas empresas, é necessário utilizar um viés mais qualitativo que quantitativo, possibilitado por meio do radar da inovação.

\subsection{RADAR DA INOVAÇÃO}

Desenvolvido a princípio por Sawhney et al. (2006), o radar propõe que a inovação seja tratada de forma holística, de modo que as organizações ponderem sobre todas as dimensões onde seja possível inovar. Isso posto, a inovação deixa de estar vinculada à simples introdução de um produto ou processo novo na organização e torna-se a criação de um valor substancial para seus clientes.

Se em Schumpeter (1988), a inovação estava ancorada em cinco perspectivas, em Sawhney et al. (2006), a inovação apresenta um conjunto 
mais amplo de possibilidades, abordando 12 dimensões: agregação de valor, cadeia de fornecimento, clientes, marca, oferta, organização, plataforma, presença, processos, rede, relacionamento e soluções. Assim, o radar da inovação apresenta a configuração descrita no Quadro 1.

\begin{tabular}{|c|c|}
\hline Dimensão & Definição \\
\hline Oferta & Desenvolvimento de novos produtos ou serviços para o mercado. \\
\hline Plataforma & $\begin{array}{l}\text { Compartilhamento de componentes, métodos ou tecnologias, tornando } \\
\text { o sistema de produção mais adaptável aos produtos ou aos serviços } \\
\text { ofertados. }\end{array}$ \\
\hline Marca & Utilização da marca para novas oportunidades de mercado. \\
\hline Cliente & Descoberta de novos segmentos ou necessidades não atendidas. \\
\hline Soluções & Combinação customizada e integrada de bens, serviços e informações. \\
\hline Relacionamento & $\begin{array}{l}\text { Formulação da experiência do cliente e sua interface com a } \\
\text { organização. }\end{array}$ \\
\hline $\begin{array}{l}\text { Agregação de } \\
\text { Valor }\end{array}$ & $\begin{array}{l}\text { Captação do valor criado por meio de fluxos de receita não explorados } \\
\text { ou da interação com clientes e parceiros. }\end{array}$ \\
\hline Processos & $\begin{array}{l}\text { Alteração das atividades realizadas na condução das operações internas } \\
\text { à empresa a fim de obter maior eficiência, melhor qualidade ou ciclo } \\
\text { mais rápido. }\end{array}$ \\
\hline Organização & $\begin{array}{l}\text { Modificações na estrutura da empresa, nas parcerias estabelecidas e no } \\
\text { papel e responsabilidade dos colaboradores. }\end{array}$ \\
\hline $\begin{array}{l}\text { Cadeia de } \\
\text { Fornecimento }\end{array}$ & Aspectos logísticos do negócio, como transporte, estocagem e entrega. \\
\hline Presença & $\begin{array}{l}\text { Novas formas de comercialização e/ou distribuição para disponibilizar } \\
\text { produtos. }\end{array}$ \\
\hline Rede & $\begin{array}{l}\text { Melhorias nos recursos de comunicação com os clientes para ampliar o } \\
\text { valor da empresa. }\end{array}$ \\
\hline
\end{tabular}

\section{Quadro 1: Dimensões do radar de inovação}

Fonte: Elaborado pelos autores, com base em Sawhney et al. (2006)

Diferentemente dos modelos originais, o radar amplia a perspectiva dos tipos de inovação, de forma que a visão não se restrinja a dimensões específicas, expandindo as possibilidades de inovação dentro de uma organização e permitindo uma mensuração mais abrangente (Paredes, Santana \& Fell, 2014). Segundo Oliveira, Cavalcanti, Brito Filho e Torres (2011) e Oliveira, Cavalcanti, Paiva Júnior e Marques (2014), o radar rompe com o sentido tradicional da inovação associada a novos produtos e norteia a medida de inovação em um sentido mais amplo, da agregação de valor à organização por um modelo sustentável, flexível e adaptado à característica do setor.

Para Gomes e Kruglianskas (2009), a estrutura de gestão exerce um papel fundamental no desempenho inovador da empresa. Segundo os autores, as grandes empresas têm um elevado grau de inovação em 
virtude, dentre outros fatores, de sua estrutura de gestão que Ihes confere vantagens. Visto que as análises até o presente propostas na literatura têm como objeto de estudo as consequências da estrutura de gestão sobre a inovação de empresas de grande porte, resta saber, portanto, quais fatores de gestão empresarial estariam relacionados ao grau de inovação obtido pelas MPEs, conforme aqui pesquisado.

\subsection{GESTÃO EM MICRO E PEQUENAS EMPRESAS}

Diante da dificuldade de mensurar o nível de gestão de empresas MPE por meio das metodologias convencionais, baseadas em seus resultados quantitativos, o Sebrae, o Movimento Brasil Competitivo (MBC) e a Gerdau, com apoio técnico da Fundação Nacional da Qualidade (FNQ), desenvolveram um questionário de autoavaliação que "permite um diagnóstico objetivo e a medição do grau de maturidade da gestão com base no Modelo de Excelência da Gestão (MEG)" (Sebrae, 2015, p. 10).

Segregados em oito critérios, o MEG possibilita a avaliação do grau de maturidade da gestão, verificando processos gerenciais e resultados organizacionais e proporcionando a compreensão do mercado e do cenário onde a organização atua e com os quais se relaciona (FNQ, 2013). Os critérios abordados pelo MEG e os fatores avaliados em cada dimensão estão expostas no Quadro 02.

\begin{tabular}{|l|l|}
\hline \multicolumn{1}{|c|}{ Critérios } & \multicolumn{1}{c|}{ Definição } \\
\hline Liderança & $\begin{array}{l}\text { Papel da liderança na empresa e investimento no desenvolvimento gerencial. Observa a } \\
\text { formulação da missão, a comunicação e o compartilhamento de informações com os } \\
\text { colaboradores e como as inovações são promovidas. }\end{array}$ \\
\hline $\begin{array}{l}\text { Estratégias e } \\
\text { Planos }\end{array}$ & $\begin{array}{l}\text { Processo de definição das estratégias e dos planos futuros e sua comunicação aos } \\
\text { colaboradores, estabelecimento de indicadores, metas e planos de ação. }\end{array}$ \\
\hline Clientes & $\begin{array}{l}\text { Identificação das necessidades e expectativas dos clientes potenciais e atuais, divulgação dos } \\
\text { produtos/serviços, relacionamento com os clientes. }\end{array}$ \\
\hline Sociedade & $\begin{array}{l}\text { Analisa as práticas de gestão da empresa em relação à sociedade no atendimento às exigências } \\
\text { legais, aspectos ambientais e sociais. }\end{array}$ \\
\hline Informações e & $\begin{array}{l}\text { Informações necessárias à execução das atividades da empresa e à tomada de decisão. } \\
\text { Observa como é realizado o compartilhado de conhecimento entre dirigentes e colaboradores e } \\
\text { a utilização de informações comparativas na análise do desempenho. }\end{array}$ \\
\hline Pessoas & $\begin{array}{l}\text { Definição das funções na empresa e suas responsabilidades, seleção e capacitação das } \\
\text { pessoas, riscos e perigos associados ao trabalho e práticas de bem-estar. }\end{array}$ \\
\hline Processos & $\begin{array}{l}\text { Processos principais do negócio da empresa, relacionamento dos fornecedores com a empresa } \\
\text { e a gestão financeira realizada. }\end{array}$ \\
\hline Resultados & $\begin{array}{l}\text { Resultados apresentados pela empresa, relativos aos clientes, aos colaboradores, aos } \\
\text { processos principais do negócio e aos resultados financeiros. }\end{array}$ \\
\hline
\end{tabular}

Quadro 2: Critérios de gestão baseados no MEG

Fonte: Elaborado pelos autores, baseado em Sebrae (2015) 
Dada sua flexibilidade, o modelo torna-se útil para efetuar "avaliação, diagnóstico e orientação de qualquer tipo de organização, no setor público ou privado, com ou sem finalidade de lucro, e de porte pequeno, médio ou grande" (FNQ, 2005, p. 9). Por tal motivo, vê-se como adequada a utilização do MEG para mensurar dimensões de gestão em empresas MPE.

Bento, Schmidt, Nogueira e Nogueira (2014) compatibilizaram os critérios MEG com o modelo de inovação Modelo de Excelência da Inovação (MEI), e de maneira geral encontraram forte correlação entre os modelos. Os autores verificaram que as dimensões Pessoas e Liderança são as que possuem maior relação com os elementos do modelo de inovação, o que é justificado, dado que ambas as dimensões abrangem a construção do ambiente propício à inovação por papel do líder/gestor ou de seus colaboradores. É necessário ponderar, contudo, se tal realidade faz parte do universo das MPEs, dado que a figura do líder/empreendedor costuma ser mais ativa que a de seus colaboradores, o que pode tornar a inovação um processo unidirecional.

As variáveis Clientes e Sociedade possuem relações médias com praticamente todos os elementos do MEI, o que demonstra que elas afetam o modelo de inovação, porém não de maneira decisiva. Percebe-se também que aspectos considerados na dimensão Informações e Conhecimento, por exemplo, são abordados nos estudos de Zaheer e Bell (2005), Gomes e Kruglianskas (2009) e Santa et al. (2011). Aspectos referentes à dimensão Clientes e Pessoas são considerados em Panesar e Markeset (2008) e Souza e Bastos (2009), respectivamente. E, conforme demonstrado, Sutthijakra (2011) e Damanpour e Schneider (2008) tratam da inovação considerando os papéis sociais e ambientais da organização, enquanto Cainelli et al. (2006) e Canepa e Stonemany (2007) a relacionam com fatores econômicos e financeiros.

Dado o respaldo literário, acredita-se que essas dimensões possam exercer influência no desempenho de inovação considerando-se a realidade das MPEs. Ressalta-se, todavia, que as dimensões não abordadas na literatura também podem trazer contribuições para o resultado obtido. 
Dessa forma, a análise será efetuada considerando-se as diversas possibilidades de relação até efetuar-se a delimitação de um modelo.

\section{PROCEDIMENTOS METODOLÓGICOS}

A captação dos dados de gestão e inovação aqui analisados foi realizada por meio do programa Agente Locais de Inovação (ALI), que surgiu de uma parceria entre o Sebrae e o Conselho Nacional de Desenvolvimento Científico e Tecnológico (CNPq). Dessa forma, por meio do programa ALI, foram obtidos os dados referentes ao segmento de restaurantes e outros estabelecimentos de serviço de alimentação e bebidas, por meio da aplicação de questionários a empresários de 88 MPEs na região metropolitana do Recife.

No radar da inovação do Sebrae, as 12 dimensões de inovação propostas por Sawhney et al. (2006) são abordadas por 30 constructos, transformados em perguntas e agrupados nas 12 categorias.

Como neste trabalho analisa-se a inovação de forma geral, a pontuação obtida por cada dimensão foi utilizada para medição do Grau de Inovação (GI) de cada MPE, dado por:

$$
G I_{i}=\left(\sum_{k=1}^{13} D_{i k)}\right) / k
$$

em que:

$\mathrm{GI}_{\mathrm{i}}$ é o grau de inovação da empresa i;

$D_{i k}$ é o valor da dimensão da inovação k para a empresa i;

;

k é o número de dimensões de inovação consideradas no modelo.

Para este trabalho, o Grau de Inovação tem se mostrado uma medida efetiva, uma vez que é realizada a análise de um único setor, dessa forma, não é necessário efetuar o cálculo do Grau de Inovação Setorial (GIS) como realizado por Oliveira et al. (2011) e Oliveira et al. (2014), dado que a heterogeneidade dos setores não afeta os resultados da pesquisa.

Por sua vez, o desempenho da gestão é mensurado por meio de 37 constructos agrupados nos oito critérios (dimensões de gestão) apresentadas no MEG. O diagnóstico realizado entende que cada dimensão possui uma contribuição diferente para a gestão da empresa, assim, 
assumem-se pesos específicos para cada dimensão, de forma que as pontuações registradas de uma mesma alternativa, mas de diferentes dimensões, possuem valores distintos.

O valor obtido por cada dimensão de gestão é dada pela seguinte fórmula:

$$
D_{g i}=\sum_{c=1}^{6} \frac{P_{g} P_{a}}{c}
$$

em que:

$\mathrm{D}_{\mathrm{gi}}$ é o valor de cada dimensão de gestão g para a empresa i; ;

$\mathrm{P}_{\mathrm{g}}$ é o peso atribuído a cada dimensão de gestão g;

$\mathrm{P}_{\mathrm{a}}$ é o peso atribuído à alternativa considerada;

;

c é o número de constructos referentes a cada dimensão de gestão.

Como se pretende prever o grau de inovação de uma empresa em função das variáveis de gestão, o modelo desenvolvido foi uma regressão linear múltipla, em que $O G_{i}$ é o termo dependente e $D_{g i}$ os termos independentes, expresso a princípio pela equação a seguir.

$$
G I_{i}=\alpha+\beta_{1} D_{1 i}+\beta_{2} D_{2 i}+\beta_{3} D_{3 i}+\beta_{4} D_{4 i}+\beta_{5} D_{5 i}+\beta_{6} D_{6 i}+\beta_{7} D_{7 i}+\beta_{8} D_{8 i}+\varepsilon_{i}
$$

em que:

a é o intercepto da equação;

$\beta_{i}$ representa os coeficientes da equação;

;

$\varepsilon_{\mathrm{i}}$ representa o termo de erro.

Os cálculos do Grau de Inovação e das Dimensões de Gestão foram

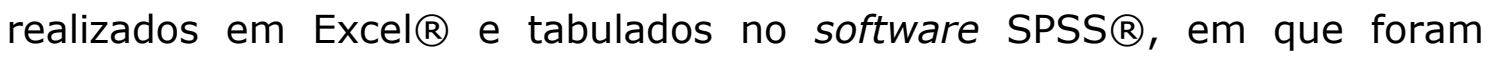
efetuadas as análises estatísticas, complementadas pelo STATA®. Para análise dos outliers, calcularam-se os resíduos padronizados e a distância de Mahalanobis, que mede os afastamentos dos valores a partir das médias das variáveis previsoras (Field, 2009), assim, excluíram-se da análise cinco itens da amostra, cujos resíduos foram superiores a 3 ou inferiores a -3 e/ou apresentaram valores discrepantes no teste de Mahalanobis. Dessa forma, a amostra totalizou com 83 empresas. 


\section{RESULTADOS}

A partir dos dados obtidos do Radar de Inovação e Diagnóstico em Gestão, efetuou-se o cálculo das variáveis consideradas no modelo, conforme exposto na metodologia, obtendo-se assim o Grau de Inovação (como variável dependente) e as nove dimensões de gestão (como as variáveis independentes) das 88 empresas pertencentes à amostra.

$\mathrm{Na}$ Tabela 01, são apresentadas as estatísticas básicas das dimensões consideradas das 83 empresas, uma vez que foram excluídos os outliers. Salienta-se que os resultados discriminados a seguir são válidos para o grupo de empresas estudadas e refletem a posição dessas empresas pertencentes à região metropolitana do Recife no período de 2013 a 2015.

Tabela 1: Estatísticas básicas

\begin{tabular}{l|r|r|c|r}
\hline & Média & $\begin{array}{r}\text { Modelo } \\
\text { padrão }\end{array}$ & $\begin{array}{c}\text { Variân } \\
\text { cia }\end{array}$ & Potencial \\
\hline Liderança & 0,8865 & $\begin{array}{r}0,4171 \\
9\end{array}$ & 0,174 & 2,50 \\
\hline $\begin{array}{l}\text { Estratégias e } \\
\text { Planos }\end{array}$ & 0,4954 & $\begin{array}{r}0,3886 \\
9\end{array}$ & 0,151 & 2,25 \\
\hline Clientes & 0,6365 & 0,2521 & 0,064 & 1,80 \\
\hline Sociedade & 1,0030 & 0,3520 & 0,124 & 2,00 \\
\hline Informações e & 0,4215 & 0,1867 & 0,035 & 1,50 \\
\hline Conhecimento & 0,5441 & 0,3574 & 0,128 & 1,80 \\
\hline Pessoas & & 4 & & \\
\hline Processos & 1,7861 & 0,5747 & 0,330 & 4,00 \\
\hline Resultado & 0,1009 & 0,2023 & 0,041 & 5,00 \\
\hline Grau de Inovação & 2,2103 & 0,2813 & 0,079 & 5,00 \\
\hline & & & & \\
\hline
\end{tabular}

Fonte: Dados da pesquisa

Para análise da média de cada dimensão, deve ser levado em consideração que cada critério possui pesos diferentes conforme a avaliação realizada pelo programa ALI. Dessa forma, a simples avaliação da média poderia levar a resultados discrepantes, uma vez que, mesmo que a empresa obtivesse pontuação máxima em critérios como Liderança e Resultado, por exemplo, as médias nessas dimensões seriam bem distintas 
dado que a pontuação máxima do critério Liderança é 2,5 enquanto o critério Resultado é 5.

Isso posto, a análise das médias será efetuada a partir da diferença entre a pontuação máxima de cada dimensão (apresentada na coluna potencial da Tabela 1). Assim, as menores divergências são verificadas nas dimensões Sociedade (Potencial - $\mu=0,997$ ), Informações e Conhecimento (Potencial - $\mu=1,0785$ ) e Clientes (Potencial - $\mu=1,1635$ ). O que expressa maior atenção na administração das pequenas empresas quanto à sociedade no atendimento a exigências legais, aspectos sociais e ambientais, uma vez que o não atendimento às exigências legais referentes a prefeitura, corpo de bombeiros e vigilância sanitária podem resultar em intervenção no estabelecimento; nas informações necessárias à execução da atividade e à tomada de decisão; e no conhecimento das necessidades e expectativas de seus clientes atuais e potenciais.

Em contrapartida, apesar de a dimensão Processos exibir média elevada, verifica-se que apresentou média abaixo de seu potencial (Potencial - $\mu=2,2139$ ), indicando um baixo comprometimento das empresas com a padronização e o controle dos principais processos de negócio. O baixo desempenho também é verificado em Estratégias e Planos (Potencial - $\mu=1,7546$ ), indicando que a condução do negócio é realizada sem a formulação de visão de futuro e o desenvolvimento de planejamento. Por fim, constatou-se que, comparativamente, os Resultados apresentados pela empresa (em termos de margem de lucro, produtividade, satisfação dos clientes, etc.) foram os que apresentaram divergências maiores (Potencial - $\mu=4,8991$ ), sinalizando, em média, um comportamento médio de baixa eficiência operacional.

Percebe-se ainda, pela análise da moda, que grande parte das empresas respondentes não possui informações suficientes para avaliar seus resultados, o que é representado pela nota zero, evidenciando a falta de controle e acompanhamento de resultados financeiros e não financeiros. Nesse sentido, segundo dados levantados pelo Sebrae-SP (2014), 47\% das empresas de pequeno porte em atividade no estado de São Paulo não observam rigorosamente receitas e despesas de seus empreendimentos. 
Quanto ao nível de inovação apresentado pelas empresas, cujo cálculo é realizado pela média aritmética das dimensões da inovação, observou-se $\mu=2,2103$ e $\sigma^{2}=0,079$, o que indica que os valores se encontram concentrados em torno da média, demonstrando que, no geral, as empresas consideradas na amostra desenvolvem inovações de maneira ocasional. Não houve registro de empresas que desenvolvem inovação sistematicamente, visto que o valor máximo obtido pela amostra foi de 2,83, muito aquém de 5,00, que indicaria tal situação.

A fim de verificar se o desempenho em uma dimensão do radar poderia afetar outra dimensão, foi realizada a correlação de Spearman (uma vez que a maioria das variáveis violou a hipótese de normalidade dos dados), efetuando a classificação dos dados em primeiro lugar e depois aplicando a equação de Pearson (Field, 2009). Conforme se observa na Tabela 2, o coeficiente de correlação indicou não existência de relacionamento significativo entre as variáveis, corroborando os resultados do teste VIF. Verifica-se ainda que todas as correlações com a variável dependente foram positivas, o que corrobora a hipótese testada neste trabalho, e indica que as variáveis independentes não são estocásticas.

Tabela 2: Correlação de Sp

\begin{tabular}{l|c|c|c|c|c|c|c|c|c}
\hline & Liderança & $\begin{array}{c}\text { Est. e } \\
\text { Planos }\end{array}$ & Clientes & Sociedade & $\begin{array}{c}\text { Info. e } \\
\text { Conhec. }\end{array}$ & Pessoas & Processos & Resultado & GI \\
\hline Liderança & 1,000 & $0,582^{* *}$ & $0,338^{* *}$ & $0,287^{* *}$ & $0,586^{* *}$ & $0,484^{* *}$ & $0,517^{* *}$ & 0,167 & $0,605^{* *}$ \\
\hline $\begin{array}{l}\text { Estr. e } \\
\text { Planos }\end{array}$ & $0,582^{* *}$ & 1,000 & $0,229^{*}$ & 0,018 & $0,480^{* *}$ & 0,203 & $0,443^{* *}$ & 0,012 & $0,416^{* *}$ \\
\hline Clientes & $0,338^{* *}$ & $0,229^{*}$ & 1,000 & 0,127 & $0,239^{*}$ & $0,259^{*}$ & $0,217^{*}$ & 0,138 & $0,322^{* *}$ \\
\hline Sociedade & $0,287^{* *}$ & 0,018 & 0,127 & 1,000 & 0,083 & $0,471^{* *}$ & $0,285^{* *}$ & 0,020 & $0,358^{* *}$ \\
\hline $\begin{array}{l}\text { Info. e } \\
\text { Conhec. }\end{array}$ & $0,586^{* *}$ & $0,480^{* *}$ & $0,239^{*}$ & 0,083 & 1,000 & $0,378^{* *}$ & $0,506^{* *}$ & 0,151 & $0,523^{* *}$ \\
\hline Pessoas & $0,484^{* *}$ & 0,203 & $0,259^{*}$ & $0,471^{* *}$ & $0,378^{* *}$ & 1,000 & $0,532^{* *}$ & 0,055 & $0,495^{* *}$ \\
\hline Processos & $0,517^{* *}$ & $0,443^{* *}$ & $0,217^{*}$ & $0,285^{* *}$ & $0,506^{* *}$ & $0,532^{* *}$ & 1,000 & 0,192 & $0,521^{* *}$ \\
\hline Resultado & 0,167 & 0,012 & 0,138 & 0,020 & 0,151 & 0,055 & 0,192 & 1,000 & 0,124 \\
\hline GI & $0,605^{* *}$ & $0,416^{* *}$ & $0,322^{* *}$ & $0,358^{* *}$ & $0,523^{* *}$ & $0,495^{* *}$ & $0,521^{* *}$ & 0,124 & 1,000 \\
\hline
\end{tabular}

** A correlação é significativa no nível 0,01 ( 2 extremidades).

* A correlação é significativa no nível 0,05 (2 extremidades).

Fonte: Dados da pesquisa

\subsection{ANÁLISE DA RELAÇÃO ENTRE GRAU DE INOVAÇÃO E DIMENSÕES DE GESTÃO}

Das dimensões de gestão analisadas, apenas Clientes, Sociedade, Informações e Conhecimento e Processos seguiram os pressupostos 
paramétricos. As demais demonstraram não haver relação linear com a variável dependente, portanto não serão incluídas no modelo de regressão linear múltipla. E, de fato, a análise da regressão, como se verá, apresentou resultados superiores quando essas variáveis não foram consideradas.

Os pressupostos da regressão linear múltipla também foram atendidos pelas dimensões inclusas no modelo. Os resíduos apresentaram distribuição normal com média zero e variância constante. O teste de Durbin-Watson indicou que os resíduos possuem correlação igual a zero e o teste de White demonstrou haver homocedasticidade dos erros, indicando que o modelo linear convencional é indicado para a análise em questão (Field, 2009).

Dessa forma, foi rodada uma regressão linear múltipla, na qual se testou o poder explicativo das diversas dimensões de gestão analisadas sobre o Grau de Inovação das MPEs. O conjunto de variáveis cujo maior poder explicativo sobre o Grau de Inovação se obteve, conforme expresso na regressão, é composto pelas variáveis independentes: Processos, Clientes, Sociedade e Informações e Conhecimento para as quais foi obtido um R² de 46,6\% para a variável dependente Grau de Inovação. Ou seja, esse composto de variáveis tem poder explicativo de $46 \%$ sobre o processo de inovação das empresas em epígrafe.

\section{Tabela 3: Resumo do modelo}

\begin{tabular}{l|c|c|c|c}
\hline Modelo & $\mathbf{R}$ & $\begin{array}{c}\mathbf{R} \\
\text { quadrado }\end{array}$ & $\begin{array}{c}\mathbf{R} \text { quadrado } \\
\text { ajustado }\end{array}$ & $\begin{array}{c}\text { Erro padrão } \\
\text { da } \\
\text { estimativa }\end{array}$ \\
\hline 1 & $0,682^{\mathrm{a}}$ & 0,466 & 0,438 & 0,2108692 \\
\hline $\begin{array}{l}\text { Nota: } \\
\text { Processos, Clientes, Sociedade, Informações e Conhecimento }\end{array}$ \\
Fonte: Dados da pesquisa
\end{tabular}

Segundo os resultados da ANOVA, a regressão é significativa ( $F=$ $17,002 \mid p$-value $=0,000)$, apresentando 78 graus de liberdade. Conforme demonstrado na Tabela 4, em que estão calculados os coeficientes das mesmas variáveis independentes pela regressão, todas elas se mostraram significativas, o que resulta no seguinte modelo: 


$$
G I_{i}=1,441+0,194 C_{i}+0,194 S_{i}+0,473 I_{i+} 0,127 P_{i}+\varepsilon_{i}
$$

em que:

$\mathrm{GI}_{\mathrm{i}}$ é o grau de inovação da empresa i;

;

$\mathrm{C}_{\mathrm{i}}$ é o valor de cada dimensão de Clientes para a empresa i;

$\mathrm{S}_{\mathrm{i}}$ é o valor de cada dimensão de Sociedade para a empresa i;

$\mathrm{I}_{\mathrm{i}}$ é o valor de cada dimensão de Informações e Conhecimentos para a empresa i;

$P_{i}$ é o valor de cada dimensão de Processos para a empresa i;

$\varepsilon_{\mathrm{i}}$ representa o termo de erro.

Tabela 4: Resultado da Regressão Linear Múltipla

\begin{tabular}{|c|c|c|c|c|c|c|c|}
\hline & \multirow{2}{*}{ Modelo } & & \multicolumn{2}{|c|}{$\begin{array}{c}\text { Coeficientes não } \\
\text { padronizados }\end{array}$} & \multirow{2}{*}{$\begin{array}{c}\begin{array}{c}\text { Coeficient } \\
\text { es }\end{array} \\
\text { padroniza } \\
\text { dos }\end{array}$} & \multirow{2}{*}{$\mathbf{t}$} & \multirow{2}{*}{ Sig. } \\
\hline & & & B & $\begin{array}{l}\text { Modelo } \\
\text { padrão }\end{array}$ & & & \\
\hline \multirow{5}{*}{1} & (Constante) & & 1,441 & 0,101 & & $\begin{array}{c}14,28 \\
2\end{array}$ & $\begin{array}{c}0,000 * \\
*\end{array}$ \\
\hline & Clientes & & 0,194 & 0,097 & 0,174 & 1,999 & $0,049 *$ \\
\hline & Sociedade & & 0,219 & 0,068 & 0,274 & 3,199 & $\underset{*}{0,002 *}$ \\
\hline & $\begin{array}{l}\text { Informações } \\
\text { Conhecimento }\end{array}$ & e & 0,473 & 0,152 & 0,314 & 3,108 & $\begin{array}{c}0,003 * \\
*\end{array}$ \\
\hline & Processos & & 0,127 & 0,049 & 0,259 & 2,579 & $0,012 *$ \\
\hline
\end{tabular}

Nota: Variável dependente: Grau de Inovação

Preditores: (Constante), Processos, Clientes, Sociedade, Informações e Conhecimento

** A correlação é significativa no nível 0,01 (2 extremidades).

* A correlação é significativa no nível 0,05 (2 extremidades).

Fonte: Dados da pesquisa

Em consonância com os resultados alcançados por Panesar e Markeset (2008), as práticas de gestão em relação aos Clientes mostraramse relevantes para a propensão das empresas a inovar, $\operatorname{com} \beta=0,174$ e $p$ value $=0,049$. Essa constatação pode ser explicada pela busca das empresas em se manterem mais competitivas e em melhor responder a crescentes níveis de exigência dos consumidores. Outro fator externo que, segundo a literatura, é relevante para a decisão de inovar das empresas constitui o aspecto Sociedade. Sutthijakra (2011) destacou a relevância da 
adaptação da empresa a aspectos contextuais, enquanto Damanpour e Schneider (2008) salientaram a inovação como possível resposta a variáveis ambientais. No mesmo diapasão, as empresas aqui estudadas demonstraram a relevância da variável Sociedade sobre o Grau de Inovação, obtendo-se $\beta=0,274$ e $p$-value $=0,002$.

A dimensão Processos mostrou-se relevante para explicar o Grau de Inovação de pequenas empresas, com $\beta=0,259 p$-value $=0,012$, que estão relacionados com a padronização de processos, relacionamento com fornecedores e gestão financeira. Os resultados aqui alcançados na análise de MPEs corroboraram as perspectivas de Neely e Hii (1998) e de MolinaPalma (2004). Em ambos os trabalhos, entende-se que a capacidade de inovação empresarial resulta da inter-relação sinérgica entre cultura organizacional, relações inter-organizacionais e dos processos internos. A influência positiva da dimensão de processos empresariais nas MPEs sobre sua atividade de inovação pode decorrer do fato de que processos gerenciais mais estruturados possibilitam ao gestor diminuir as miopias sobre as atividades e produtos da empresa, bem como melhor conhecer as expectativas e necessidades de seu público-alvo, instrumentos essenciais para desenvolver novos produtos e processos na empresa de forma eficaz, fomentando, assim, um ambiente corporativo mais propício à inovação.

Entretanto, quanto ao aspecto Informações e Conhecimentos, cujo $\beta$ $=0,314$ e $p$-value $=0,003$, os resultados aqui obtidos mostraram um comportamento inverso ao defendido por Santa et al. (2011). Uma possibilidade para o alcance de um resultado diverso talvez seja o fato de os autores terem se limitado a inquirir sobre a implementação de tecnologias da informação, enquanto, na presente pesquisa, exploram-se também aspectos relativos à obtenção e ao compartilhamento do conhecimento entre os dirigentes e colaboradores e à utilização de informações comparativas externas para a análise do desempenho da empresa.

Nesse sentido, para Gomes e Kruglianskas (2009), as fontes de conhecimento constituem um aspecto crítico para a inovação, o que confere à organização uma vantagem competitiva importante. Zaheer e Bell (2005), por sua vez, demonstram que empresas que ocupam uma posição superior 
de network e possuem conexões com fontes externas de conhecimento apresentam melhores desempenhos, uma vez que a estrutura a torna mais hábil para explorar suas capacidades internas e melhorar sua performance pelo acesso rápido ao conhecimento e à capacidade de identificar oportunidades (Gomes \& Kruglianskas, 2009), o que facilita o potencial inovador.

Embora o trabalho de Bento et al. (2014) tenha se desenvolvido no sentido de que dimensões de Liderança e Pessoas são aquelas que apresentam maior relação com os elementos que auxiliam a inovação, na presente análise, os resultados foram diversos, observando-se haver uma relação não significativa com a variável dependente. Em outros termos, a dimensão Liderança proposta pelo radar da inovação do Sebrae não tem poder explicativo sobre o Grau de Inovação das MPE em estudo, o que pode ser consequência de nestas organizações o processo de inovação tender a centrar-se unicamente na figura do líder/empreendedor e não na de todos os colaboradores. Ainda, outra possibilidade que pode reforçar esse fenômeno nas MPEs pode resultar de situações como, sem maiores incentivos, não se constrói um ambiente propenso à inovação nas MPEs, pois os colaboradores tenderiam a preferir manter sua segurança com o status quo, sem inovar e assumir os riscos de tal iniciativa.

As dimensões Pessoas e Estratégia e Planos também não apresentaram relação linear com a variável Grau de Inovação nas MPEs, contradizendo, portanto, quanto ao aspecto de gestão dos colaboradores, o observado por Gebauer et al. (2008) e seu conjunto de 15 variáveis explicativas para a inovação em empresas, sob as quais estavam envolvimento dos trabalhadores da linha de frente, distribuição da informação, constituição e educação das equipes, tecnologia de informação, organização interna; e o trabalho de Souza e Bastos (2009), cuja argumentação é que organizações com maiores níveis de inovação tendem a implementar políticas de gestão de pessoas. Pela análise das médias expostas na Tabela 1, percebe-se que, em média, as MPEs apresentam níveis de gestão de pessoas que nem sequer alcançam a metade do potencial do índice (Potencial - $\mu=1,2559$, para um potencial de até 1,8 ), 0 que indica claramente uma gestão de pessoas deficiente na mediada em 
que os processos de seleção, determinação de funções e responsabilidades são realizados de forma intuitiva, enquanto as práticas de capacitação e bem-estar são realizadas de forma esporádica.

No que se refere à variável Estratégia e Planos, observou-se um baixo desempenho médio (Potencial - $\mu=1,7546$, para um potencial máximo de 2,25). Essa falta de comprometimento das MPEs em estudo com a formulação de uma visão de futuro e o desenvolvimento de planejamento pode explicar a relação não significativa entre a dimensão Estratégias e Planos e o Grau de Inovação. Afinal, a decisão de investir em inovação é indicada na literatura como parte da estratégia de longo prazo das empresas no escopo de alcançar maiores rendimentos e valor futuro. Todavia, conforme demonstrado anteriormente, as inovações ocorrem de forma ocasional nas MPEs, o que não necessariamente está inserido em um planejamento estratégico de longo prazo.

A diferença mais importante entre a média obtida entre as empresas em exame e o seu potencial foi encontrada na dimensão Resultado (Potencial - $\mu=4,8991$, para um potencial máximo de 5,00 ). O que indica a falta de registro e acompanhamento ineficiente dos resultados relativos a clientes, colaboradores, principais processos do negócio e resultados financeiros. Embora, a literatura defenda haver uma relação de autorreforço da dimensão econômica da empresa através da inovação, segundo dados da regressão linear múltipla, os resultados da MPEs não foram significativos para o modelo. Outra possível explicação para tal resultado seriam, conforme defendido por Canepa e Stonemany (2007), sobretudo os aspectos financeiros, que impactam significativamente a inovação promovida em empresas. 


\section{CONSIDERAÇÕES FINAIS}

No presente artigo, analisaram-se oito dimensões de gestão que agrupam 37 construtos, conforme proposto no Modelo de Excelência da Gestão para 83 MPEs da região metropolitana do Recife cujas atividades, embora do mesmo segmento, são distintas. Apesar de serem estudadas oito dimensões, a utilização de apenas quatro apresentou maior poder explicativo sobre o Grau de Inovação por possuírem relação linear com a variável dependente, registrando um $\mathrm{R}$ quadrado de $46,6 \%$.

Foi constatado que o Grau de Inovação de MPEs no setor alimentício é significativamente resultante das práticas em resposta a exigências legais, aspectos ambientais e sociais; do relacionamento com o cliente até a forma de divulgação dos produtos/serviços; das informações para a tomada de decisão e do compartilhamento de conhecimento entre dirigentes e colaboradores; e de como se executam e controlam os processos principais do negócio da empresa.

Em contrapartida, diversamente do indicado por parte da literatura sobre empresas em geral, constatou-se que o Grau de Inovação no setor estudado não é influenciado significativamente pela forma como os dirigentes exercem sua liderança, a gestão de pessoas, resultados operacionais e financeiros apresentados pela empresa, tampouco pelo processo de definição de estratégia e planos de longo prazo. O que pode ser explicado pelo reduzido investimento de MPEs em treinamento e aprendizagem de seus colaboradores, pela centralização da iniciativa para inovar na pessoa do empreendedor e pela falta de cultura do planejamento de longo prazo em MPEs em geral.

Das quatro dimensões de gestão analisadas que integraram o modelo proposto, três (Sociedade, Clientes e Informação e Conhecimento) demonstraram receber uma maior dedicação por parte do administrador. Tal constatação se fez possível pela observação de esses aspectos terem apresentado a menor diferença entre o nível máximo potencial e as medias observadas para cada um. A dimensão Processos, apesar de figurar entre as maiores médias observadas, tem um valor muito aquém de seu potencial na MPEs do setor de alimentação na região analisada. Conclui-se, portanto, 
ocorrer em geral um baixo nível de padronização e controle dos principais processos desses empreendimentos. Finalmente, constatou-se que 0 desenvolvimento da inovação nas empresas em estudo foi ocasional e não sistematicamente realizado, demonstrando um potencial de incremento significativo.

Os resultados obtidos neste estudo permitem às MPEs identificar aspectos de gestão que possam - seja pela busca por inovação, seja para incrementar a eficiência empresarial - receber maior atenção e investimento, preferencialmente no escopo de obter resultados que sejam percebidos pelos clientes ou consumidores de produtos e serviços, de forma diferenciada com evolução sustentável. É necessário destacar que os resultados aqui alcançados se limitam às empresas e ao setor estudado, pois, para uma análise que permita generalização dos resultados, é necessário tomar uma amostra representativa daquela região pesquisada.

Todavia, o presente estudo representa uma contribuição no campo da inovação, sobretudo no que se refere à análise em micro e pequenas empresas em razão da carência de dados e pesquisas nesta área. Dada a importância da inovação como fator de competitividade nas organizações, os resultados aqui demonstrados apontam os determinantes para sua viabilidade, o que pode auxiliar gestores e pesquisadores no estabelecimento de ações e desenvolvimento de políticas públicas favoráveis ao desenvolvimento da inovação organizacional.

A busca pela compreensão da influência dos diversos prismas da gestão empresarial, por fim, mostra-se figurar como um campo propício para pesquisas dada a diversidade de setores com suas características particulares, que pode propiciar variedades de modelos explicativos para a propensão à inovação das empresas. 


\section{REFERÊNCIAS}

Bachmann, D. L., \& Destefani, J. H. (2008). Metodologia para estimar o grau das inovações nas MPE. Curitiba: Bachmann \& Associados.

Barras, R. (1986). Towards a theory of innovation in services. Research Policy, 15(4), 161-173.

Bento, M. H. S., Schmidt, A. S., Nogueira, C. U., \& Nogueira, H. M. C. M. (2014, maio/ago.). Sistema integrado de gestão: compatibilização do Modelo de Excelência em Gestão (MEG) com o Modelo de Excelência em Inovação (MEI). Desafio Online, 2(2), 674-692.

Bignetti, L. P. (2006). Gestão de tecnologia e inovação: uma análise de autores, vertentes teóricas e estratégias metodológicas predominantes em trabalhos apresentados nos Encontros da ANPAD. Anais do Encontro Nacional da Associação de Pós-Graduação e Pesquisa em Administração, 30, Salvador, BA, Brasil.

Cainelli, G., Evangelista, R., \& Savona, M. (2006, maio). Innovation and economic performance in services: a firm-level analysis. Cambridge Journal of Economics, 30(3), 435-458.

Canepa, A., \& Stoneman, P. (2007). Financial constrains to innovation in UK: evidence from CIS2 and CIS3. Oxford Economic Papers, 60(4), 711730.

Chen, Y., Podolski, E., \& Veeraraghavan, M. (2015, December). Does managerial skill facilitate corporate innovative success? Journal of Empirical Finance, 34, 313-326.

Chen, J., Tsou, H., \& Huang, A. (2009). Service delivery innovation: antecedents and impact on firm performance. Journal of Service Research, 12(1), 36-55.

Damanpour, F., \& Schneide, R. M. (2008). Characteristics of innovation and innovation adoption in public organizations: assessing the role of managers. Journal of Public Adminstration Research and Theory, 19(3), 495-522.

Exame. (2013). Pesquisa mapeia inovação nas pequenas empresas. Recuperado em 14 de novembro, 2015, de http://exame.abril.com.br/pme/noticias/pesquisa-mapeia-inovacao-naspequenas-empresas.

Field, A. (2009). Descobrindo a estatística usando o SPSS (2a ed.). porto Alegre: Artmed.

Fundação Nacional da Qualidade - FNQ. (2005). Rumo à excelência 2006: critérios para avaliação do desempenho e diagnóstico organizacional. São Paulo: FNQ. 
Fundação Nacional da Qualidade - FNQ. (2013). Modelo de excelência da gestão. São Paulo: FNQ. Recuperado em 10 de novembro, 2015, de http://fnq.org.br/e-book_MEG.pdf.

Gebauer, H., Krempi, R., Fleisch, E., \& Frideli, T. (2008). Innovation of product-related services. Managing Service Quality, 18(4), 387-404.

Gomes, C. M., \& Kruglianskas, I. (2009). A influência do porte no comportamento inovador da empresa. Revista de Administração e Inovação, 6(2), 5-27.

Grynzpan, F. (2007). O investimento privado em P\&D pela indústria de transformação no Brasil. Anais do Seminário Internacional sobre Avalliação de Políticas de Ciência, Tecnologia e Inovação, Rio de Janeiro, RJ, Brasil.

Holmstrom, B. (1989). Agency costs and innovation. Journal of Economical Behavior and Organizations, 12(3), 305-327.

Meza, M. L. F. G. (2009). O processo de gestão de inovação alinhado à estratégia de competição numa micro e pequena empresa: o caso da Cristófoli Biossegurança. Anais do Encontro de Estudos de Estratégia, 15, Recife, PE, Brasil.

Molina-Palma, M. A. (2004). A capacidade de inovação como formadora de valor: análise dos vetores de valor em empresas brasileiras de biotecnologia. Tese de Doutorado, Faculdade de Economia, Administração e Contabilidade da Universidade de São Paulo: SP, Brasil.

Neely, A., \& Hii, J. (1998). Innovation and business performance: a literature review. Government Office of Easten Region. Cambridge: University of Cambridge.

Oliveira, M. R. G., Cavalcanti, A. M., Brito Filho, J. P., Torres, D. B., \& Mattos, S. M. (2011). Grau de inovação setorial: uma abordagem a partir do radar de inovação. Anais do Encontro Nacional de Engenharia de Produção, 31, Belo Horizonte, MG, Brasil.

Oliveira, M. R. G., Cavalcanti, A. M., Paiva Júnior, F. G., \& Marques, D. B. (2014, janeiro/março). Mensurando a inovação por meio do grau de inovação setorial e do característico setorial de inovação. Revista de Administração e Inovação, 11(1), 115-137.

Organizations for Economic Cooperation and Development - OECD. (2005). Manual de Oslo (3a ed.). Rio de Janeiro: Finep/OCDE,

Panesar, S., \& Markeset, T. (2008). Development of a framework for industrial service innovation management and coordination. Journal of Quality in Maintenance Engineering, 14(2), 177-193.

Paredes, B. J. B., Santana, G. A., \& Fell, A. F. A. (2014). Um estudo da aplicação do Radar da Inovação: o grau de inovação organizacional em 
uma empresa de pequeno porte do setor metal-mecânico. Navus Revista de Gestão e Tecnologia, 4(1), 76-88.

Paswan, A., D'Souza, D., \& Zolfagharian, M. (2009). Toward a contextually anchored service innovation typology. Decision Sciences, 49(3), 513540 .

Perin, M., Sampaio, C., \& Hooley, G. (2007). Impacto dos recursos da empresa na performance de inovação. Revista de Administração de Empresas - RAE, 47(4), 46-58.

Porter, M. E. (1989). Vantagem competitiva: criando e sustentando um desempenho superior (17a ed.). Rio de Janeiro: Campus.

Porter, M. E. (1993). A vantagem competitiva das nações. Rio de Janeiro: Campus.

Póvoa, L. M. C., \& Monsueto, S. E. (2012, junho). Tamanho das empresas, interação com universidades e inovação. Revista de Economia, 37(4), 924.

Prajogo, D. I. (2006). The relationship between innovation and business performance: a comparative study between manufacturing and service firms. Knowledge and Process Management, 13(3), 218-225.

Quadros, R. (2005). Padrões de gestão da inovação tecnológica em empresas brasileiras: as diferenças por tamanho, nacionalidade e setor de negócios (Relatório de Pesquisa), Campinas, SP, Universidade Estadual de Campinas.

Santa, R., Scavarda, A., Zhao, F., \& Skoko, H. (2011, junho). Managing the operational effectiveness in services using technological innovation. International Journal of e-Business Management, 5(1), 16-32.

Sawhney, M., Wolcott, R. C., \& Arroniz, I. (2006, April). The 12 different ways for companies to innovate.MIT Sloan Management Review, 47(3), 75-81.

Schumpeter, J. A. (1988). A teoria do desenvolvimento econômico. São Paulo: Nova Cultural.

Serviço Brasileiro de Apoio às Micro e Pequenas Empresas. (2015). Questionário de autoavaliação: o talento brasileiro com apoio total para se desenvolver. São Paulo: Sebrae.

Souza, J. J., \& Bastos, A. V. B. (2009, maio-junho). Programas de qualidade de vida no trabalho em contextos diferenciados de inovação: uma análise multivariada. Revista Eletrônica de Administração - REAd, 15(2).

Sutthijakra, S. (2011). Managing service subsidiaries through an innovation perspective: a case of standard interpretation in multinational hotels. The Service Industries Journal, 31(4), 545-558. 
Symeonidis, G. (1996, January). Innovation, firm size and market structure: schumpeterian hypothesis and some new themes. OECD Economics Department Working Papers 161.

Zaheer, A., \& Bell, G. G. (2005, Sepetember). Benefiting from network position: firm capabilities, structural holes and performance. Strategic Management Journal, 26(9), 809-826. 